\title{
The History of Mathematics: A Source-Based Approach (Volumes 1 \& 2)
}

\section{Reviewed by William T. Ross}

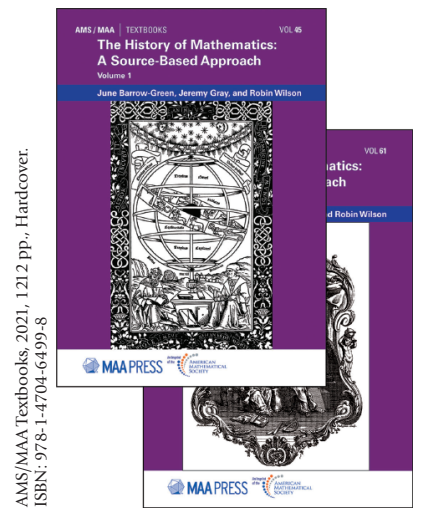

The History of Mathematics:
A Source-Based Approach (Volumes 1 \& 2)
by June Barrow-Green, Jeremy Gray,
and Robin Wilson
In many mathematics depart-
ments, a history of mathematics
course can be difficult to im-
plement. Most departments do
not have an expert historian of
mathematics and often rely on
well-intentioned, but possibly
untrained, faculty to offer this
type of course. Such faculty might feel overwhelmed when choosing the topics, readings, and assignments. What makes most standard undergraduate mathematics courses run smoothly, especially for an instructor inexperienced with the topic, is a solid book that gives faculty sufficient guideposts and students a carefully written and engaging book they can lean on whenever explanations get past them during lectures. As a textbook on the history of mathematics, a good book should also refer to original sources, so as to not devolve into silly stories about eccentric mathematicians, as well as provide commentary to give the student some guidance on how to approach and interpret an original text. In addition, a good history of mathematics text should balance an erudite seriousness, like T. L. Heath's classic books on various

William T. Ross is the Roger Francis and Mary Saunders Richardson Chair in Mathematics at the University of Richmond. His email address is wross arichmond.edu.

Communicated by Notices Book Review Editor Stephan Ramon Garcia.

For permission to reprint this article, please contact: reprint-permission aams.org.

DOI: https://dx.doi.org/10.1090/noti2377 topics and translations of Greek mathematics [2], with a lively written tone as to draw the student into the subject, whatever their mathematical background.

Barrow-Green, Gray, and Wilson have written a twovolume set on the history of mathematics that not only meets all of the above requirements for being a useful textbook, but goes one step further and provides well-researched commentary on how historians approach their subjects as well as how they consider the voracity of their sources. This current two-volume set is, in a way, a more textbook version of the well-known, and well-used, The History of Mathematics: A Reader [1], by John Fauvel and one of the current authors Jeremy Gray. In this current set, students get to see how historians approach their subjectsespecially when the subject is mathematics. For example, in Chapter 3 of Volume 1, when discussing Proclus's historical summary of the ancient Greek mathematics of Thales, Pythagoras, and Euclid, the authors point out that despite the authoritative tone of the "Proclus summary," one must keep in mind that Proclus penned it some 750 years after these events. They clarify this point even further when they write "This is roughly our distance from the signing of the Magna Carta." Arresting statements like this encourage the reader to carefully consider the difficult historical questions concerning the intentions of the writer, their sources, as well as their accuracy. This experience might be jarring for undergraduate mathematics students who are used to learning a collection of well-established and seemingly noncontroversial theorems. These books aim to give these theorems and their authors a proper backstory but leave open the debate that some of these sources could be unreliable and are open to further classroom and essay discussion. Mathematics is part of history and is subject to controversaries, biases, and inaccuracies, just like any other subject.

The first volume covers a selection of topics ranging from the 20,000-year-old Ishango bone, possibly used as 
a computational device by Paleolithic mathematicians, all the way to early seventeenth century European mathematicians with sprightly, but serious, writing that does not get overly fussy about the technical details. Along with the above early counting methods, this volume begins with the mathematics of Egypt and Mesopotamia and provides examples of how these early mathematicians struggled with concepts, notation, and communication, as all mathematicians do. They do an excellent job of showing the student how these ancients understood mathematics, how they communicated it, why they communicated it, and to whom they communicated it.

The authors next move on to the Greeks and open their discussion with a wonderful reading from Plato's Meno where Socrates takes a young student through the initial false starts, and ending with the correct solution of the doubling the square problem (given a square, construct another square with twice the area). This reading nicely sets up the development of the proof-based mathematics of the Greeks and how the Greek mathematics grew out of the earlier work of the Egyptians. The authors cover the big names of Thales, Pythagoras, Euclid, Apollonius, Archimedes, along with readings from this period. Also included in these chapters are a treatment of Euclid's Elements, the classical straightedge and compass problems, the conics, and the brilliant results of Archimedes. This is followed by a section on the Hellenistic mathematics of Ptolemy and Diophantus.

The next two chapters cover the mathematics of India, China, and the Islamic world. At the beginning of this discussion, the authors spend a considerable amount of time pointing out the difficulties in covering the mathematics of this period and of these cultures. There is the obvious issue of what we mean by the very terms of Indian, Chinese, and Islamic mathematics. Each of these individual cultures were diverse, had moving borders, and did not always share a common language amongst their populations. Then of course there are the meaningless contests of who discovered what first and the potential biases of knowing that certain traditions used proof-based mathematics while others, prima facie, did not (not quite true) and are thus considered somehow, and falsely, deficient. Into these known complexities, the authors proceed to cover the origins of the Indian decimal system, the work of Aryabhata (astronomy, geometry, a Pythagorean theorem, estimates of pi), and the beginnings of trigonometry; the mathematics of the Chinese (The Nine Chapters on Mathematical Procedures - systems of linear equations, the Pythagorean theorem, approximations of pi, the Chinese remainder theorem); and the mathematics of the Islamic (Arab, Persian) world (the algebra of Muhammad ibn Musa al-Khwarizmi and Omar Khayyam and the geometry of al-Abbas ibn Said al-Jawhari and Thabit ibn Qurra which struggled to make sense of the parallel postulate).
The next several chapters cover European mathematics from medieval times to the early seventeenth century. The authors do an excellent job of discussing the nonmathematical historical events of that time, the Black Death being of special importance, and the reasons and types of exchanges between the Islamic world and Christian Europe. They give a detailed presentation of the mathematics of that period (Fibonacci, Cardano, Viète, etc.) but also of the infrastructure that played an important role in supporting European mathematics, "the rise of the universities." The authors cover these infrastructure discussions to illustrate that mathematics is not always done by the lonely genius working in isolation, but flourishes because of an infrastructure that facilitates good communication within a community of adequately supported scholars. They end their European tour with the mathematics supporting the astronomy of Copernicus, Kepler, Galileo (interesting mathematics all by itself) and then come full circle to their earlier chapters on the Greeks when they cover Descartes's "challenge" to Euclid.

The second volume connects with the standard undergraduate curriculum of calculus, linear algebra, real and complex analysis, number theory, and applied mathematics and would make a great textbook for a capstone course for mathematics majors. Compared to the first volume, where the emphasis was on ancient mathematics and attribution becomes difficult, this second volume tends to focus on the mathematicians themselves. Indeed, the material is more modern and modern scientists demand credit for their results!

The first two chapters of Volume 2 cover the types of research done by Descartes, Fermat, Cavalieri, and Wallis who struggled with the concepts of volume, area, tangents, and normals, all of which would be addressed with the development of the calculus by Newton and Leibniz. Here the authors emphasize that not only did the concepts of the calculus (derivatives, integrals, infinite series) need to be properly developed, but the notation as well. Newton and Leibniz are each given top billing and there are beautifully selected readings to get the reader into the minds of both. Since Newton was the giant, there is a greater emphasis on him as evidenced by the thorough treatment given of the Principia.

As befitting the book's philosophy of going beyond the development of mathematical ideas, Chapter 6 covers "The spread of the calculus" and the big names every undergraduate student knows well such as Euler, L' Hopital, Taylor, and the Bernoulli brothers. What is important here are the treatises that helped spread these ideas to a wider audience. The notation and language of calculus became standardized which helped spread the word as well. Of course, making calculus (real analysis) rigorous was taken up by Cauchy. Indeed, the arresting questions of Bolzano provided the need for rigorous definitions of continuity and convergence. 
Intermediate chapters cover the players, and the development of group theory, number theory, Fourier series, and applied mathematics. The two chapters that are worth emphasizing in this review are Chapters 13 and 17. Many students have the false idea that mathematicians work on great ideas, write them up, and they become famous. Part of this is true. However, there are cultural aspects of mathematics (certain societies devote more resources to it than others) and politics and institutions come into play. Chapter 13 covers the infrastructure of European mathematics with a survey on the institutes and journals that played a crucial role in the success of mathematics. In fact, one can probably make the case that the infrastructure of mathematics is as important as mathematics itself. Students need to know this and Chapter 13 covers it well.

Though undergraduate students tend to focus on the tangible aspects of mathematics, it is important for them to see, in Chapter 17, that mathematicians are philosophers at times and grapple with foundational questions (What are properties of the real number line? How do we understand infinity? What is a set?). What makes this chapter more enlightening than a standard treatment of this subject is that the material is presented with the very words of the mathematicians such as Cantor, Dedekind, and Peano, who struggled with these ideas.

To further make the case that these books would make excellent texts for a history of mathematics course, the authors include a final chapter in each volume containing various types of exercises. What is a mathematics textbook without plenty of exercises! This final exercise chapter begins with some sage advice on writing a college-level essay, including proper use and citation of sources, along with a stern warning about plagiarism.

The "Sample Exercises" give some thoughts on what a good student should be considering when given an openended question. For example, a student is asked to write a survey on the range of Greek mathematics. Anticipating that a potential lazy student might merely parrot back the account from the text (or the internet!), the authors give the student some guidance, in the form of leading questions, to give some idea of what they might want to consider for their essay. For example, some of the questions are "Was there a core to Greek mathematics, built upon a recognition of the key importance of proof and the power of geometrical reasoning? Or is that an artefact, built partly on accidents of transmission and partly on our prejudices for theory over application and tidy sources over messy ones? So, your answer, and a very different one written by someone else with whom you disagree, may be equally valid and receive an equal mark. Disagreeing is part of being a historian."

There are three basic types of exercises in these books. The first type is one where students are presented with a historical quote and are asked to extract and contextualize its historical and mathematical value. For example, a student is presented with the statement and proof from Euclid's
Elements (VI.13) "To two given straight lines to find a mean proportional." A good student will be asked to understand the proof, engage with these connections (the existence of two mean proportionals connects with the doubling the cube problem), and do a little background research to further explore the significance of all this.

The second type of exercises relates to specific chapters where the student is asked to reexamine the readings and commentary in the book and support their argument. For example, there is the question: "Fermat is sometimes described as being simultaneously 'ancient' and 'modern'. By making reference to examples of his work, describe how accurate you consider this opinion to be."

The final type of exercises are very broad and do not apply to any particular text but instead relate to a topic a student found particularly interesting. An example of this type is "Some mathematicians have worked in almost complete isolation, others in large mathematical communities. By discussing at least three examples, consider how being part of a wider community helps or hinders a mathematician to lead a creative life."

The authors have written readable and well-researched texts, the first of which I'm currently using for a course that focuses on the Greeks. The writing is excellent. The tone is serious. The pedagogical thought that went into this will be much appreciated by both instructors and their students. As further evidence that these books are designed so students can thrive, they have been field tested in a year-long course at the Open University. Indeed, as the authors comment in their introduction "No-one should have left school with too little mathematics to follow this book. But for those who were cut off from mathematics because they could not appreciate why it was done, and who could not connect to its excitements, we hope that this historical account offers a rewarding way in."

\section{References}

[1] John Fauvel and Jeremy Gray, The history of mathematics: A reader, Macmillan/Open University, 1992.

[2] T. L. Heath, A history of Greek mathematics, Oxford at the Clarendon Press, Oxford, 1921.

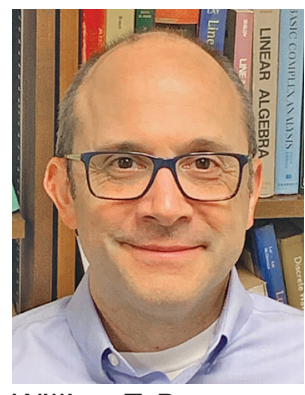

William T. Ross

\section{Credits}

Photo of William T. Ross is courtesy of William T. Ross. 